\title{
How common is self-treatment in non gonococcal urethritis?
}

\author{
E M Carlin, S E Barton
}

\begin{abstract}
Objective-To assess the prevalence of self-treatment in men with new episode non gonococcal urethritis (NGU).

Method-Three hundred consecutive men with new episode NGU attending an open access genitourinary medicine clinic were interviewed using a semistructured questionnaire. Details of treatment used before attending the clinic were obtained.

Results-Thirty (10\%) men had used treatment, for an average of 7 days, prior to the clinic attendance. Agents used included antibiotics (11), savlon or iodine (4), vitamin tablets (4), cisapride (2), local anaesthetic gel (2), antiseptic cream (2), cod liver oil (1), ferrous sulphate (1), naproxen (1), clotrimazole cream (1) and a poultice (1). Over $70 \%$ of the men reporting antibiotic use had selfmedicated and in one case a fixed drug eruption had ensued.

Conclusion-A significant number of men with NGU self-treat. In addition to the adverse effects of such treatment and the potential effect on culture tests, clinic attendance may be postponed, the use of appropriate therapy delayed, and sexual contacts will remain at risk. Early attendance for treatment and contact tracing is essential and should be actively promoted.
\end{abstract}

(Genitourin Med 1995;71:400-401)

Keywords: Urethritis; Non gonococcal; Self treatment

\section{Introduction}

Non gonococcal urethritis (NGU) may be caused by a wide variety of agents predominantly Chlamydia trachomatis, Ureaplasma urealyticum or Mycoplasma genitalium although in some cases no specific cause can be identified. ${ }^{12}$ Sexual transmission of Chlamydia trachomatis to female partners may cause serious morbidity with the development of pelvic inflammatory disease or infertility. Strategies to control the infection and its complications should therefore centre on detecting and treating the infection as soon as possible with assiduous tracing of sexual contacts. ${ }^{3}$

A community study has shown that $34 \%$ of women with urinary symptoms or vaginal discharge would treat themselves prior to consulting a doctor with $17 \%$ buying proprietary medicines. ${ }^{4}$ In women attending a genitourinary medicine (GUM) clinic we have previously shown that $56 \%$ of women have used a self-administered remedy prior to attendance with over $50 \%$ tolerating symptoms for longer than ten days. ${ }^{5}$

The frequency of self-treatment in men with NGU is unknown. It will however, result in a delay in presentation for appropriate treatment and the initiation of contact tracing which has important public health implications.

\section{Objective}

To assess the prevalence of self-treatment before attending a GUM clinic in men with new episode NGU.

\section{Subjects and method}

Three hundred consecutive men attending an open access GUM clinic with new episode, microscopically confirmed NGU were enrolled to the study. Microscopic confirmation consisted of the identification on a Gram stained urethral smear, of greater than 5 pus cells per high powered field together with the absence of Gram-negative diplococci. The men were interviewed, using a semi-structured questionnaire, to obtain details of treatment used prior to attending the clinic.

\section{Results}

The men were aged 19-59 years and were predominantly heterosexual. Over $60 \%$ had a previous history of sexually transmitted disease (STD), approximately half of which was cited as NGU.

Thirty $(10 \%)$ men had used treatment, for an average of seven days, prior to their clinic attendance (table). Over $70 \%$ of the men reporting antibiotic use had self-medicated by therapies from their own medicine cabinet or

Agents used by men with NGU prior to attending a clinic

\begin{tabular}{ll}
\hline Agent & Number of men using agent \\
\hline Antibiotics & 11 \\
Savlon or iodine & 4 \\
Vitamin tablets & 4 \\
Cisapride & 2 \\
Local anaesthetic gel & 2 \\
Antiseptic cream & 2 \\
Cod liver oil & 1 \\
Ferrous sulphate & 1 \\
Naproxen & 1 \\
Clotrimazole cream & 1 \\
Mustard poultice & 1 \\
\hline
\end{tabular}

St Stephen's Clinic,
Chelsea and
Westminster Hospital,
London SW10 9NH
E M Carlin
S E Barton
Accepted for publication
22 May 1995


via friends, only two using antibiotics which had previously been prescribed for NGU. In all cases the antibiotic was either inappropriate or inadequate and in one man a fixed drug eruption had ensued.

There were no significant differences in age range, sexuality and previous STD or NGU history in men who had self-medicated before attending the clinic compared with untreated men.

\section{Discussion}

Self-medication has become increasingly common in the community among a wide range of individuals and alternative therapy may be used without the physicians knowledge. . $^{6-9}$

We ascertained that a significant number of men with NGU self-medicated, mostly from their own or their friends' stored medication, with at least one adverse reaction. We did not perform urine screening for antimicrobials as our study was aimed at examining attenders' reports of self-medication. However, it is likely that the $10 \%$ prevalence of self-treatment identified is an underestimate as others have shown that $60 \%$ of clinic attenders with antimicrobial agents in the urine deny antibiotic ingestion. ${ }^{10}$ Hence, incorporation of a urine screen for antimicrobials would have added further weight to the study. Our GUM clinic is an open access service where clients can be seen for assessment on the day of presentation. Yet, in men who self-medicated there was an average delay of seven days before presenting at the clinic and obtaining appropriate therapy and during this time their sexual contacts remained at risk.

Whilst individuals should be encouraged to participate fully in their own health care early GUM clinic attendance rather than selftreatment for NGU is essential and should be actively promoted. Only then can an accurate diagnosis with effective treatment and contact tracing be instituted with the opportunity to offer screening for other STDs and the promotion of sexual health.

1 Stephanik M, Rychna K, Valkoun A. Microbial causative agents of male urethritis. F Hyg Epidemiol Microbiol Immunol 1992;36:111-8.

2 Horner PJ, Gilroy CB, Thomas BJ, Naidoo ROM, Taylor-Robinson D. Association of Mycoplasma genitalium with acute non-gonococcal urethritis. Lancet 1993;342:582-5

3 Taylor-Robinson D. Chlamydia trachomatis and sexually transmitted disease. What do we know and what shall we do? BMF 1994;308:150-1.

4 Jolleys JV. The reported prevalence of urinary symptoms in women in one rural general practice. $\mathrm{Br} \mathcal{F}$ Gen Pract 1990;40:335-7.

5 Russell JM, Barton SE, Lawrence AG. Self-medication by women attending a genitourinary medicine clinic. Int $f$ women attending a genitourinary medicine clinic. Int $\mathcal{f}$

6 Habeeb GE Jr, Gearhart JG. Common patient symptoms: patterns of self-treatment and prevention. $\mathcal{F}$ Miss State Med Assoc 1993;34:179-81.

7 Stoller EP, Forster LE, Portugal S. Self-care responses to symptoms by older people. A health diary study of illness behavior. Med Care 1993;31:24-42.

8 Richards S, Thornhill D, Roberts H, Harries U. How many people think they have hay fever, and what they do about it. Br 7 Gen Pract 1992;42:284-6.

9 Kassler WJ, Blanc P, Greenblatt R. The use of medicinal herbs by human immunodeficiency virus-infected patients. Arch Intern Med 1991;151:2281-8.

10 Gordon SM, Mosure DJ, Lewis J, Brown S, McNagny SE, Schmid GP. Prevalence of self-medication with antibiSchmid GP. Prevalence of self-medication with antibi-
otics among patients attending a clinic for treatment of sexually transmitted diseases. Clin Infect Dis 1993;17: 462-5. 